\title{
Detection of the Adulteration of Traditional Alcoholic Beverages by the Separation and Determination of Alprazolam, Chloralhydrate and Diazepam Using Reversed-Phase High-Performance Liquid Chromatography
}

\author{
R. Nageswara Rao, ${ }^{\dagger}$ P. Parimala, Sara Khalid, and S. Naseeruddin Alvi \\ HPLC Group, Division of Analytical Chemistry, Indian Institute of Chemical Technology, \\ Tarnaka, Hyderabad-500 007, India
}

\begin{abstract}
A simple, rapid and reliable reversed-phase high-performance liquid chromatographic method for the separation and determination of psycotropic substances viz., alprazolam, chloral hydrate and diazepam in traditional alcoholic beverages, such as toddy, has been developed. Separation was accomplished using a reversed-phase $\mathrm{C}_{18}$ column with water-methanol-acetic acid $(35: 65: 0.1 \mathrm{v} / \mathrm{v} / \mathrm{v})$ as a mobile solvent and a photo-diode array detector at $210 \mathrm{~nm}$. The limits of detection of alprazolam, chloral hydrate and diazepam were determined to be $0.8,4.5$ and $0.4 \mu \mathrm{g}$, respectively. The validity of the method was checked by analyzing nearly 200 samples collected from different outlets by enforcement authorities, and the extent of adulteration was determined.
\end{abstract}

(Received April 28, 2003; Accepted September 25, 2003)

\section{Introduction}

Food is essential not only for the sustenance of life, but also for the maintenance of good health. However, contaminated or adulterated food is a major source of human illness, and results in a serious loss of nutrition. ${ }^{1}$ Thus, the quality and safety of food assumes great significance in the promotion of optimum health. Toddy is one of the alcoholic drinks traditionally prepared by the fermentation of sap or exudate collected by slicing off the tip of unopened flowers of either a coconut or a palm tree. ${ }^{2}$ It is a social and local drink produced and consumed throughout Asia, particularly India, Sri Lanka and Bangladesh. Due to the increase in consumer demand and the shortage of coconut or palm tree, it has often been diluted and adulterated with chemical substances, such as chloral hydrate (CHL), diazepam (DIA) or alprazolam (ALP) belonging to nonbarbiturates and benzodiazepines, respectively. ${ }^{3,4}$ These drugs are generally considered to be dangerous when consumed along with alcohol, and have proved to be fatal poisonings according to the recent analysis of forensic toxicological investigations conducted by Koski et al. ${ }^{5}$ Further, it was reported that female drinkers are at increased risk to abuse benzodiazepines when compared to men. ${ }^{6}$ A case of alprazolam poisoning present with coma and respiratory depression in a young girl was also reported. ${ }^{7}$ These psycotropic substances have a sedative action and are added illicitly to toddy to increase its potency. The adulterated toddy is prohibited for consumption under the laws of excise, which necessitates the development of suitable analytical methods for the simultaneous detection and determination of the levels of psycotropic substances in fermented food and alcoholic beverages.

Fujiwara test, which gives an intense red color by the action

† To whom correspondence should be addressed.

E-mail: rnrao@iict.ap.nic.in of pyridine in the presence of an alkali, is generally used to identify CHL in toddy. ${ }^{8-10}$ A number of assays have shown CHL to be genotoxic ${ }^{11}$ and hepato-carcinogenic in male mice. ${ }^{12}$ Due to its widespread potential for human exposure, US FDA has declared CHL as a priority chemical for an in-depth toxicological evaluation. As a part of this program, a capillary gas chromatography with electron-capture detection was developed for the determination of CHL and its metabolites in blood plasma of mice and rats. ${ }^{13}$ Two liquid-chromatographic methods based on reversed-phase and anion-exchange mechanisms were also reported in the literature. ${ }^{14}$ However, both of the methods are preceeded by the derivatization of CHL with dansylhydrazine and o-(4-nitrobenzyl)hydroxylamine, followed by detection using UV and suppressed conductivity detectors. A spot test using Dragendorffs reagent containing bismuth nitrate and potassium iodide in an acetic acid medium produces an orange-red color, indicating the presence of DIA. ${ }^{15}$ The colorimetric determination of DIA in pharmaceutical preparations was described by Baggi et al. ${ }^{16}$ However, these tests are not specific because similar colors are produced by a number of organic compounds containing halogens. Squarewave voltammetric studies at hanging mercury drop electrodes for the determination of 1,4-benzodiazepines over a wide range of concentrations have been conducted. ${ }^{17}$ Husain et al. have studied the differential pulse polarograms of CHL and DIA in $0.1 \mathrm{M} \mathrm{KCl}$ in $50 \%$ ethanol as a supporting electrolyte, and tried to estimate their levels quantitatively in toddy. ${ }^{18} \mathrm{~A}$ micellar liquid chromatographic procedure for the determination of benzodiazepines in serum has been reported. ${ }^{19}$

Beaulieu et al. have developed a liquid-chromatographic method using a cyano column to determine ALP and its related substances in bulk drugs. ${ }^{20}$ LC-ESI-MS and LC-MS-MS for determination of benzodiazepines in hair and plasma samples of psychiatric patients were used extensively. ${ }^{21,22}$ Spectrofluorimetric and liquid chromatographic methods for the determination of photodegradation products of ALP were also 
<smiles>Cc1nnc2n1-c1ccc(Cl)cc1C([18OH])=NC2</smiles>

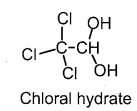<smiles>CN1C(=O)CN=C([C@H](O)CO)c2cc(Cl)ccc21</smiles>

Fig. 1 Chemical structures of the adulterants studied in the present investigation.
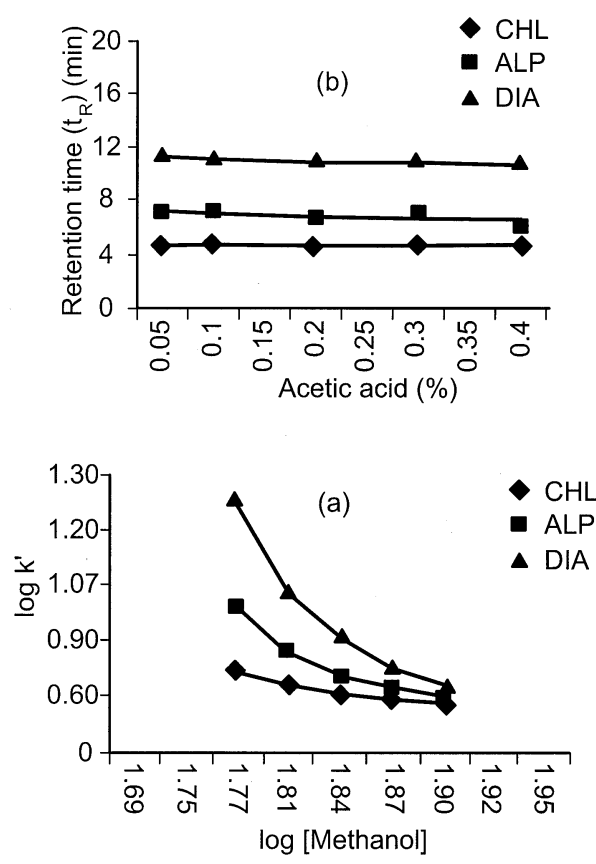

Fig. 2 Effect of concentration of (a) methanol and (b) acetic acid on the retention of CHL, ALP and DIA.

reported..$^{23,24}$ A gas chromatography coupled with ion trap tandem mass spectrometry was used to determine 22 benzodiazepines in biological matrices. ${ }^{25}$ A thorough literature search has revealed that methods for the simultaneous detection of CHL, DIA and ALP either in toddy or fermented foods have not been reported. In the present paper, we describe a simple, rapid and reliable reversed-phase HPLC method for simultaneous separation, identification and determination of CHL, DIA and ALP in fermented alcoholic beverages of toddy using a $\mathrm{C}_{18}$ column with water-methanol-acetic acid (35:65:0.1 $\mathrm{v} / \mathrm{v} / \mathrm{v}$ ) as a mobile solvent and a photo-diode array detector at $210 \mathrm{~nm}$ at ambient temperature.

\section{Experimental}

\section{Materials and reagents}

All reagents were of analytical-grade unless stated otherwise. Glass-distilled water was deionized using a Nanopure II D 3700 cartridge (Barnsted, USA). HPLC-grade methanol (Spectrochem, Mumbai, India) was used. ALP, CHL and DIA (BDH, Poole, UK) were used as reference standards.

\section{Apparatus}

A high-performance liquid chromatograph (Shimadzu, Kyoto, Japan) with a $20 \mu \mathrm{l}$ loop, LC-10Atvp pump, SPD-10Avp UVVisible spectrophotometric detector and SCL-10Avp system controller was used. A Symmetry $\mathrm{C}_{18}$ column $(25 \mathrm{~cm} \times 4.0 \mathrm{~mm}$

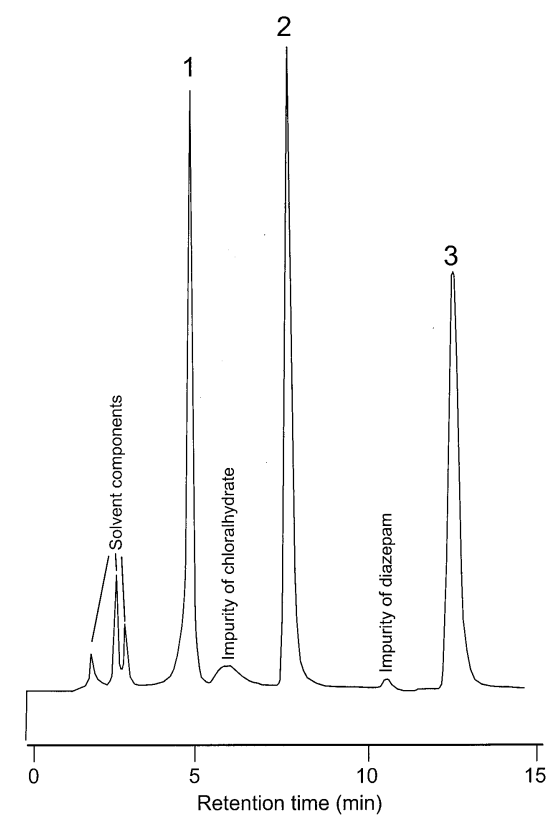

Fig. 3 Typical HPLC chromatogram of a synthetic mixture containing 1, CHL $(42 \mu \mathrm{g}) ; 2$, ALP $(2.6 \mu \mathrm{g})$; and 3, DIA $(2.3 \mu \mathrm{g})$.

i.d.; particle size, $5 \mu \mathrm{m}$ ) was used for separation. The chromatograms and the integrated data were recorded with an Intel Pentium III HP-computer system.

\section{Chromatographic conditions}

The mobile phase was water-methanol-acetic acid (35:65:0.1 $\mathrm{v} / \mathrm{v} / \mathrm{v})$. The analysis was carried out under isocratic conditions at a flow rate of $1 \mathrm{ml} / \mathrm{min}$ at room temperature $\left(27^{\circ} \mathrm{C}\right)$. Chromatograms were recorded at $210 \mathrm{~nm}$.

\section{Analytical procedure}

Samples of toddy were filtered through a 0.45 micron aqueous nylon membrane and $20 \mu \mathrm{l}$ of the filtrate was injected onto the chromatograph. Synthetic mixtures containing ALP, CHL and DIA and the samples were analyzed under identical conditions. The presence of ALP, CHL and DIA was determined by comparing the retention times with that of the standards and their quantities were estimated from the areas of the respective peaks.

\section{Results and Discussion}

The chemical structures of ALP, CHL and DIA are shown in Fig. 1. The present study was aimed at developing of a simple and rapid chromatographic system capable of eluting, resolving and detecting ALP, CHL and DIA in adulterated traditional alcoholic drink such as toddy. In our preliminary experiments, the three substances were subjected to separation by RP-HPLC on a Symmetry $\mathrm{C}_{18}$ column with methanol-water-acetic acid as an eluent. To determine the optimum separation conditions for a synthetic mixture containing ALP, CHL and DIA, the influence of the separation parameters, such as the concentration of methanol and acetic acid in the eluent on their retention, was studied. Methanol was used as an organic modifier of the mobile phase. The dependence of the retention factor $\left(k^{\prime}\right)$ of the compounds under investigation on the content of methanol and acetic acid is shown in Fig. 2. When the 


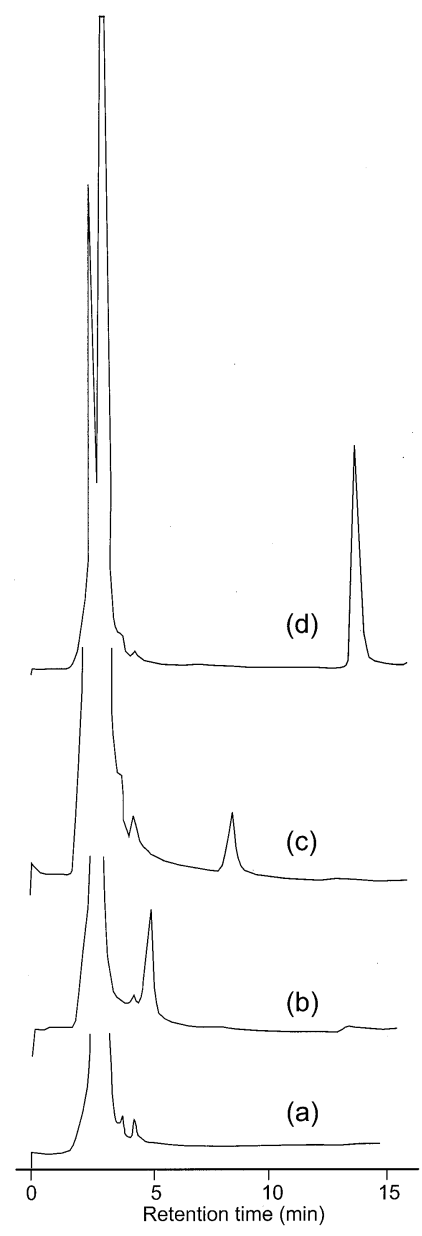

Fig. 4 Typical HPLC chromatograms of toddy, (a) without adulteration and containing, (b) CHL, (c) ALP and (d) DIA.

concentration of methanol was $65 \%$ and acetic acid $0.1 \%$, all of the compounds were eluted and separated from one another. Figure 3 shows the HPLC chromatogram of a synthetic mixture containing CHL, ALP and DIA eluted at 4.65, 7.12 and 11.22 min, respectively. It can be seen from Fig. 3 that the compounds were well separated ( $R_{\mathrm{s}(\mathrm{CHL} / \mathrm{ALP})} 2.74$ and $R_{\mathrm{s} \text { (ALP/DIA })}$ 4.31) under the conditions used. The peaks were identified by injecting individual authentic compounds. The peaks were resolved with excellent symmetry and reproducibility. In another attempt, a more-polar solvent, like acetonitrile, was tried instead of methanol. The peaks were eluted at 3.31, 4.32 and $6.38 \mathrm{~min}$, corresponding to CHL, ALP and DIA, respectively. However, it was found that the resolutions were comparatively less $\left(R_{\mathrm{S}(\mathrm{CHL} / \mathrm{ALP})} 2.02\right.$ and $\left.R_{\mathrm{s}(\mathrm{ALP} / \mathrm{DIA})} 2.57\right)$, therefore methanol was chosen as one of the chief components of the mobile phase. Further, the effect of the temperature on the retention behavior of the compounds under study was also evaluated at 40 and $50^{\circ} \mathrm{C}$. As the column temperature was increased from ambient conditions, the compounds were found to be less retained on the column with a significant loss in resolution between the peaks. Thus, the ambient conditions were found to be more suitable for the present study.

Standard mixtures containing known amounts of ALP, CHL and DIA were prepared and analyzed by HPLC. The accuracy of the method was determined by the standard addition technique. The subsequent addition of small amounts of the analytes was accurately reflected in their peak areas. The

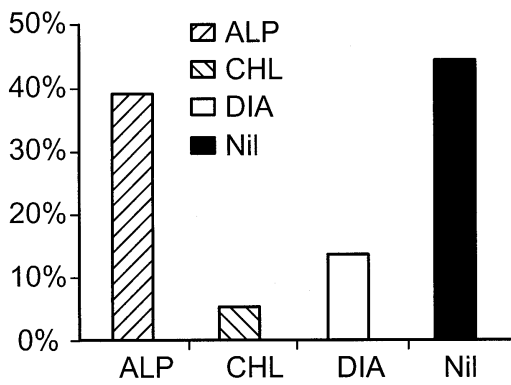

Fig. 5 Bar diagram depicting the percentage of adulteration of toddy with different substances.

measured amounts agreed well with the actual values within $2.97 \%$. Known amounts of ALP, CHL and DIA were added to toddy to yield concentrations in the range of $0.01-1.0 \mathrm{mg} / \mathrm{ml}$ and used for calibration. The linearity between the mass and the integral responses was found to be quite good. The standard curves for ALP, CHL and DIA were found to be linear with regression coefficients $\left(r^{2}\right)$ of $0.9935,0.9958$ and 0.9974 , respectively. The precision of the analysis was assessed by five replicate analyses of toddy containing ALP, CHL and DIA in the concentration range of $0.01-1.0 \mathrm{mg} / \mathrm{ml}$. The variations were then calculated. The response of a series of five injections of $10 \mu \mathrm{g}$ of ALP, CHL and DIA in toddy resulted in a relative standard deviation in the ranges of 2.5-3.6 and 2.4-3.9 for the retention time $\left(t_{\mathrm{R}}\right)$ and peak area $\left(i_{\mathrm{p}}\right)$, respectively. When the UV detector was set at 0.005 AUFS the limits of detection for ALP, CHL and DIA with a signal to noise ratio of 4.0 were determined to be $0.8,4.5$ and $0.4 \mu \mathrm{g}$, respectively.

The quality of toddy samples suspected to be adulterated with ALP, CHL and DIA were thoroughly analyzed by HPLC. The typical chromatograms of samples containing ALP, CHL and DIA are shown in Fig. 4. The presence of the adulterants in the referred samples has been confirmed not only by comparisons of retention times with those of standards, but also by on-line UV spectra obtained by PDA. Alternatively, eluent fractions corresponding to the three peaks were collected, enriched and subjected to elctrospray ionization-mass spectrometry (ESI-MS) for further confirmation. The molecular ions of 163, 308 and 284 have indicated the presence of CHL, ALP and DIA, respectively. Thus, ALP, CHL and DIA were found to be present in some of the toddy samples analyzed by HPLC. However, the degree of adulteration varied significantly. The relative response factors were determined and used to quantify the levels of adulterants in different samples of toddy. Nearly 200 samples collected by the prohibition and excise authorities have been analyzed by the proposed method. About 120 samples were found to be adulterated with the psycotropic substances studied in the present investigation. A bar diagram showing the percentage of adulteration with ALP, CHL and DIA is shown in Fig. 5.

In conclusion, the proposed HPLC method for detecting APL, CHL and DIA in adulterated samples of toddy is simple, rapid and easily adaptable for quantitative determinations. It may be used as an alternative to the spot tests that are currently being used to detect the adulterants qualitatively. It is reliable and convenient not only for quality assurance, but also forensic investigations. 


\section{Acknowledgements}

The authors wish to thank Dr. K. V. Raghavan, Director, Indian Institute of Chemical Technology, Hyderabad for his keen interest in our studies and kind permission to communicate the results for publication (IICT Publication No. 030110).

\section{References}

1. A. Madhavan, J. Oil. Chem. Assoc. Ind., 1998, 30, 181.

2. P. Fellows, Traditional Foods, Processing for profit, IT Publ, 1997, www.itdg.org/html/technical enquiries.

3. H. J. Moller, J. Clin. Psycopharmacol., 1999, 19, 2 S.

4. S. V. Argyropoulos and D. J. Nutt, Neuropsychopharmacol., 1999, 9, 407.

5. A. Koski, I. Ojanpera, and E. Vuori, Alcohol Clin Exp Res., 2002, 26, 956.

6. S. M. Evans and F. R. Levin, Behav Pharmacol., 2002, 13, 427.

7. N. R. Rathod, Ind. J. Med. Sci., 2001, 55, 218.

8. K. Fujiwara, Sber. Abh naturf. Ges. Rostock., 1916, 6, 33.

9. M. S. Prasad and M. S. Subbarao, J. Food. Sci. Technol., 1976, 13, 339.

10. S. S. Kamat, V. P. Barre, and H. S. Mahal, Analyst, 1972, 97, 877 .

11. S. Haworth, T. Lawlor, K. Mortelmans, W. Spack, and E. Zeiger, Environ. Mutagen. Suppl., 1983, 1, 3.
12. M. H. George, T. Moore, S. Kilburn, G. R. Olson, and A. B. DeAngelo, Toxicol. Pathol., 2000, 28, 610.

13. T. C. Schmitt, J. Chromatogr. B. Anal. Technol. Biomed. Life Sci., 2002, 780, 217.

14. M. C. Bruzzoniti, E. Mentasti, C. Sarzanini, and E. Tarasco, J. Chromatogr. A, 2001, 920, 283.

15. K. A. Ambade and S. K. Meghal, Analyst, 1981, 106, 1237.

16. T. R. Baggi, S. N. Mahajan, and G. R. Rao, J. Assoc. Off. Anal. Chem., 1975, 58, 875.

17. M. M. D. Santos, V. Famila, and M. L. Goncalves, Anal. Bioanal. Chem., 2002, 374, 1074.

18. S. Husain, P. Raviprasad, and N. S. Swamy, Ind J. Tech., 1991, 29, 362.

19. M. E. C. Peiro, D. Bose, A. M. Dominguez, M. G. Agusti, and J. E. Romero, J. Chromatogr. B. Anal. Technol. Biomed. Life Sci., 2002, 780, 241.

20. N. Beaulieu, S. J. Graham, R. W. Sears, and E. G. Lovering, J. AOAC Int., 1992, 75, 801.

21. T. Toyooka, Y. Kumaki, M. Kanbori, M. Kato, and Y. Nakahara, J. Pharm. Biomed. Anal., 2003, 30, 1773.

22. R. Kronstrand, I. Nystrom, M. Josefsson, and S. Hodgins, J. Anal. Toxicol., 2002, 26, 479.

23. N. S. Nudelman and C. G. Cabrera, J. Pharm. Biomed. Anal., 2002, 30, 887.

24. N. S. Nudelman and C. G. Cabrera, J. Pharm. Sci., 2002, 91, 1274.

25. S. Pirnay, I. Ricordel, D. Libong, and S. Bouchonnet, J. Chromatogr. A, 2002, 954, 235. 